\title{
Review of approaches and methods to assess the comparative efficiency of technological processes and production
}

\author{
D.I. Kovalev1, ${ }^{*}$, M.F. Kozlova ${ }^{2}$, O.I. Olshevskaya ${ }^{3}$, T.P. Mansurova ${ }^{4}$ \\ ${ }^{1}$ Krasnoyarsk State Agrarian University, Krasnoyarsk, Russian Federation \\ ${ }^{2}$ Reshetnev Siberian State University of Science and Technology, Krasnoyarsk, Russian \\ Federation \\ ${ }^{3}$ Siberian Federal University, Krasnoyarsk, Russian Federation \\ ${ }^{4}$ Krasnoyarsk Science and Technology City Hall of the Russian Union of Scientific and \\ Engineering Association, Krasnoyarsk, Russian Federation
}

*E-mail: kovalev.dw7@gmail.com

\begin{abstract}
The article provides an overview of approaches and methods to assess the comparative efficiency of technological processes and production. It is known that the most common methods are those that assess production based on the analysis of the states of control objects, as components of production, taken into account in the automated control system, or analysis of the course of the technological process. It is shown that efficiency is the most general, defining property of any purposeful activity, which from the cognitive point of view is revealed through the category of the goal and is objectively expressed by the degree of goal achievement, taking into account the cost of resources and time. Increasing the efficiency of the enterprise is carried out using methods of analyzing the structures of enterprises, which allow, with the transparency of the structure and organization of production, to carry out accurate and high-quality monitoring of the state of the objects of the enterprise and their management. The analysis of comparative efficiency is an important component of production processes. The results of the analysis allow you to debug the work of the enterprise already at the initial stages of reducing the efficiency of its functioning. An important task is to find a mathematical basis for analyzing the efficiency of enterprises. The paper discusses parametric and nonparametric methods for assessing comparative efficiency. The main difference between parametric methods is the econometric estimation of parametric functions in the study of the interaction of costs and resources. The authors pay attention to the nonparametric DEA method, which, due to its characteristics, has a number of advantages and features. The method allows you to obtain a comprehensive performance indicator for each enterprise, and can also take into account multiple input and output variables. These features of the DEA method make it possible to successfully apply it in determining the indicator of the comparative efficiency of enterprises.
\end{abstract}

Key words: comparative efficiency, automated control system, enterprise, modeling, analysis 


\title{
Обзор подходов и методов к оценке сравнительной эффективности технологических процессов и производств
}

\author{
Д. И. Ковалев ${ }^{1, *}$, М.Ф. Козлова ${ }^{2}$, О.И. Ольшевская ${ }^{3}$, Т.П. Мансурова ${ }^{4}$ \\ ${ }^{1}$ Красноярский государственный аграрный университет, Российская Федерация, \\ г. Красноярск \\ ${ }^{2}$ Сибирский государственный университет науки и технологий имени академика \\ М. Ф. Решетнева, Российская Федерация, г. Красноярск \\ ${ }^{3}$ Сибирский федеральный университет, Российская Федерация, г. Красноярск \\ ${ }^{4} \mathrm{OУ}$ «ККДНиТ», Российская Федерация, г. Красноярск \\ *E-mail: kovalev.dw7@gmail.com
}

\begin{abstract}
Аннотация. В статье дается обзор подходов и методов к оценке сравнительной эффективности технологических процессов и производств. Известно, что наиболее распространены методы, которые дают оценку производств, исходя из анализа состояний объектов управления, как составных частей производства учитываемых в АСУП или анализа хода технологического процесса. Показано, что эффективность - это наиболее общее, определяющее свойство любой целенаправленной деятельности, которое с познавательной точки зрения раскрывается через категорию цели и объективно выражается степенью достижения цели с учетом затрат ресурсов и времени. Повышение эффективности работы предприятия осуществляется при помощи методов анализа структур предприятий, которые позволяют при прозрачности структуры и организации производства осуществлять точное и качественное слежение за состоянием объектов предприятия и управление ими. Анализ сравнительной эффективности является важной составляющей процессов функционирования производства. Результаты анализа позволяют отлаживать работу предприятия уже на первоначальных этапах снижения эффективности его функционирования. Важной задачей является поиск математической основы анализа эффективности предприятий. В работе рассматриваются параметрические и непараметрические методы оценки сравнительной эффективности. Основным отличием параметрических методов является эконометрическая оценка параметрических функций при исследовании взаимодействия затрат и ресурсов. Авторы уделяют внимание непараметрическому методу DEA, который, в силу своих характеристик, обладает рядом преимуществ и особенностей. Метод позволяет получать комплексный показатель эффективности для каждого предприятия, а также может учитывать множественные входные и выходные переменные. Эти особенности метода DEA позволяют успешно применить его при определении показателя сравнительной эффективности предприятий.
\end{abstract}

Ключевые слова: сравнительная эффективность, АСУП, предприятие, моделирование, анализ

\section{1. Введение}

Разработка эффективно функционирующих автоматизированных систем управления технологическими процессами (АСУ ТП) и производствами (АСУП) немыслима без формализации основных этапов проектирования. При этом постановки математических задач, возникающих при организации проектирования и сравнении вариантов проектных решений, 
так или иначе связаны с задачами моделирования, оптимизации, выбора вариантов [1]. Известный польский специалист по методологии проектно-конструкторских разработок В. Гаспарский [2] еще в прошлом веке писал о том, что современные методологии науки в ответ на вопрос: «Что является основой проектирования?» отвечают, что основой являются критерии эффективности, выведенные из утверждений теории оптимизации. Стремление к эффективным, а не к любым допустимым решениям глубоко пронизывает современное проектирование. Методы сравнительного анализа эффективности АСУ ТП и АСУП выступают и как средства организации и модельно-алгоритмического обеспечения самого процесса проектирования, и как инструментарий подсистемы мониторинга в составе АСУП. Немаловажную роль в определении и построении подсистемы мониторинга играет выбор базовой модельно-алгоритмической концепции в виде метода оценки эффективности, как основы системы реагирования на изменения эффективности в организационнотехнологическом комплексе предприятия [3].

В работе [4] приведены наиболее распространенные методы, которые могут использоваться для оценки производств, исходя из анализа состояний объектов управления, как составных частей производства, учитываемых в АСУП, или анализа хода технологического процесса. Используемыми методами оценки и оптимизации состояния объекта управления являются: метод наименьших квадратов, метод максимального правдоподобия, метод байесовых оценок [5]. Качество управления объектом можно оценить определением математического ожидания условной вероятности [4].

Метод ранжирования [4] используется для оценки состояния объектов управления в том случае, когда необходимо контролировать параметры, ранее измерявшиеся качественно. Им приписывают некоторые количественные оценки, то есть вводят ранжирование параметров. Допускаемая при этом производительность выбора количественных оценок, затрудняет управление объектом в адаптивных системах. Чтобы унифицировать процесс ранжирования различных качественных параметров, можно использовать принцип нормированного ранжирования по какому-либо априорно принятому закону [6].

Приведенные методы рассматривают в основном оптимальность хода технологического процесса. В работе [3], в частности, анализируются методы оценки эффективности сложных производственных систем, таких как перерабатывающие производства.

\section{2. Моделирование структур предприятий в АСУП}

С целью оптимизации управления производством и возможного решения ряда проблем, связанных с охватом производственных систем и повышением уровня их управляемости, в середине 60-х годов Американское общество управления производством и запасами (APICS) 
сформулировало ряд принципов, по которым предлагалось строить модели предприятия и основных производственных процессов в них. Впервые эти принципы были применены для решения задач управления материальными запасами предприятия и получили название концепции MRP (Material Requirements Planning - планирование материальных ресурсов), основные положения которой перечислены ниже:

- модель производственного процесса описывается как поток взаимосвязанных заказов;

- при выполнении заказов учитываются ограничения ресурсов;

- обеспечивается минимизация производственных циклов и запасов;

- заказы снабжения и производства формируются на основе заказов реализации и производственных графиков;

- движение заказов увязывается с экономическими показателями;

- выполнение заказа завершается к тому моменту, когда он необходим.

Иерархическая структура АСУ технологического процесса подразумевает [7]:

- поток команд направлен от верхнего уровня к нижнему;

- нижний отвечает верхнему по его запросам.

На этапе синтеза модели в состав ее параметров и структур требуется вводить дополнительные элементы (избыточность), которые при непосредственном использовании позволят управлять качеством, снизят чувствительность модели и соответствующих показателей качества к изменениям состава, структуры и содержания исходных данных [8].

При исследовании таких систем при разработке проектов их организационнотехнологического развития необходимо учитывать их многоступенчатость. Математические модели могут быть использованы как для оценки функционирования организационнотехнологических систем, так и для прогнозирования их развития, что особенно важно в условиях оценки стратегического управления [9].

Дадим понятие модели и системы. Система представляет собой совокупность взаимосвязанных и взаимодействующих частей, выполняющих некоторую полезную работу. Частями (элементами) системы могут быть любые комбинации разнообразных сущностей, включающие людей, информацию, программное обеспечение, оборудование, изделия, сырье или энергию (энергоносители). Система - это множество элементов, находящихся в отношениях и связях друг с другом, образующих определенную целостность, единство. Модель - искусственный объект, представляющий собой отображение (образ) системы и ее компонентов. Считается, что М (модель) моделирует А (объект), если М отвечает на вопросы относительно А. Модель описывает то, что происходит в системе, как ею управляют, что она преобразует, какие средства использует для выполнения своих функций и что производит [10]. 
Одной из возможностей моделирования производственной системы предприятия является алгоритмизация. Алгоритмизация технологических процессов представляет собой разработку математического описания поведения системы. В алгоритмах реализации модели процесса, как правило, выделяются программные блоки реализации отдельно детерминированной и стохастической частей модели [4]. Поэтому математическим обеспечением являются все методы, приемы, стандартные алгоритмы, облегчающие и ускоряющие логическое проектирование предприятия в АСУП [8]. К таким методам можно отнести:

- методы исследования поведения технологических процессов и построения математических моделей;

- приемы структурной теории алгоритмов;

- программное обеспечение для автоматизации программирования;

- стандартное программное обеспечение, используемое как составная часть программ управления.

Более того, в публикации В.В. Биличенко [9] приводится предположение о том, что все производственные системы формально подобны между собой. Это подобие основано на таких важнейших атрибутах систем как: организация, управление, технология, социальнопсихологические отношения, правовая регуляция, экологические требования и многое другое. Производственные системы отличаются одна от другой масштабом, а также видами предметов, которые потребляются и изготавливаются, энергетическими и информационными ресурсами.

Входы-элементы, которые поступают в систему, могут рассматриваться как ресурсы, которые обеспечивают внедрение в неё мероприятий разных направлений организационнотехнологического развития. Так продукты других систем, например, сырье, техника, виды продукции и другие обусловливают элементы-входы других подсистем. На этой основе может быть построена иерархическая система факторов - показателей эффективности использования ресурсов системы (см. рисунок 1), которые обусловливают уровень ее конечных результатов [9]. 


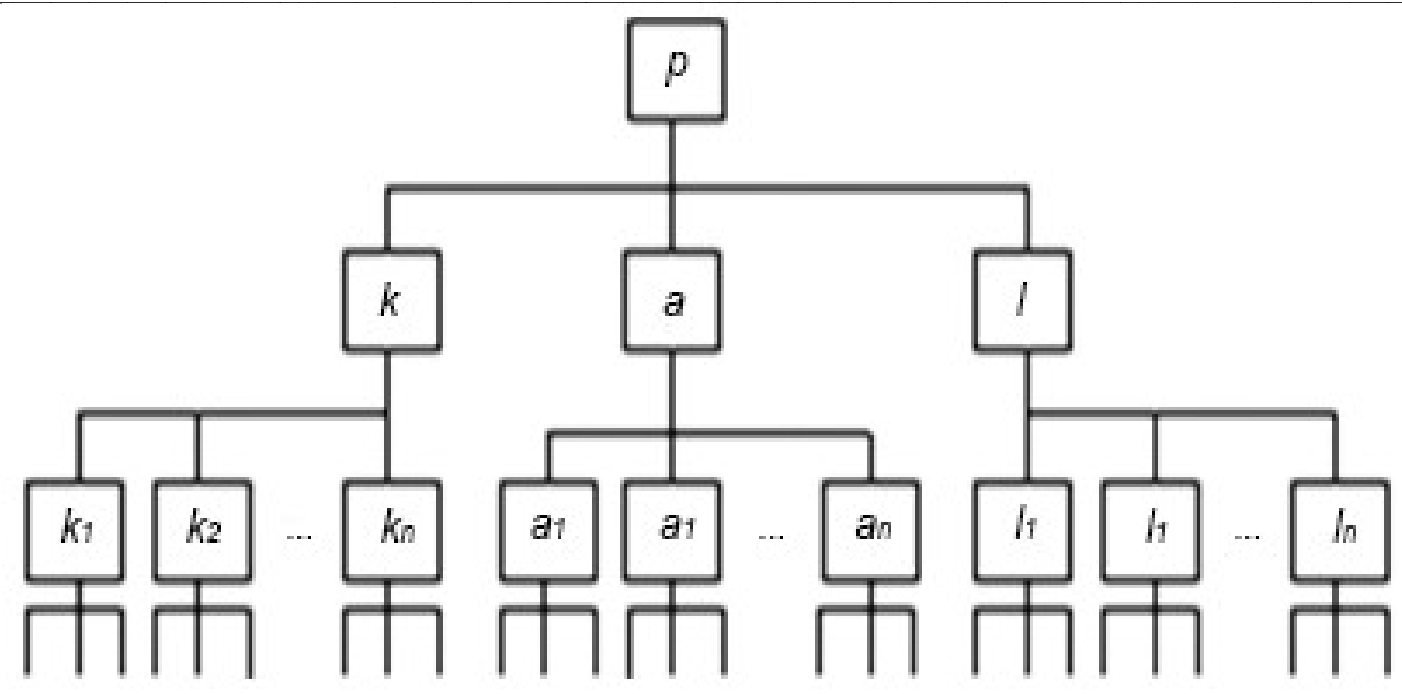

Рисунок 1. Иерархическая система факторов производства.

На рисунке 1: $p$ - показатель, который выражает конечный результат функционирования системы, это может быть объем перевозок, рост производительности труда, прибыль, рентабельность и другие; $k$ - показатель эффективности использования основных фондов; $a$ - показатель эффективности использования предметов труда (запасных частей, материалов и другие); $l$ - показатель эффективности использования труда.

\section{3. Анализ функционирования предприятий с целью повышения его эффективности}

Прежде чем разобрать понятие эффективности предприятия, познакомимся с понятием «эффективности» вообще. Эффективность - это наиболее общее, определяющее свойство любой целенаправленной деятельности, которое с познавательной точки зрения раскрывается через категорию цели и объективно выражается степенью достижения цели с учетом затрат ресурсов и времени $[11,12]$.

Эффективность может рассматриваться как функция от цели. Эффективность комплексная характеристика потенциальных и (или) реальных результатов использования системы с учетом степени соответствия этих результатов целям, стоящим перед системой [8]. Выступает как индикатор развития, его важнейший стимул, становится целевым ориентиром управленческой деятельности, направляет эту деятельность в русло обоснованности, необходимости, оправданности и достаточности.

Эффективность предприятия определяют сопоставлением результатов от функционирования предприятия и затрат всех видов ресурсов, необходимых для его поддержания и развития. Критерий эффективности предприятия определяют на множестве (системе) показателей, каждый из которых описывает одну из сторон рассматриваемой 
системы. В зависимости от используемого математического аппарата критерий может быть выражен в виде целевой функции или порядковой меры, устанавливающей упорядоченную последовательность сочетаний показателей. При определении результатов от функционирования системы управления в АСУП задают универсальную систему обобщенных показателей, таких, как оперативность (своевременность), устойчивость, качество управления и другие. Используемые показатели должны быть развернуты применительно к характеристикам конкретной АСУП (например: оперативность - вероятностно-временные характеристики элементов процесса управления; устойчивость - показатели надежности, помехозащищенности) [7].

Целесообразные варианты построения предприятия выбирают путем балансирования показателей приращения эффективности Э, получаемой за счет поддержания в работоспособном состоянии или совершенствования предприятия, и затрат $Q$. Математически эту задачу формируют в виде:

$\max Э$ при $Q=$ const;

или в виде обратной задачи:

$\min Q$ при Э= const.

К основным частным показателям, характеризующим экономическую эффективность предприятий [7,13], относят:

- годовую экономию (годовой прирост прибыли);

- снижение издержек производственно-хозяйственной деятельности на объектах управления;

- повышение производительности труда;

- экономию по видам ресурсов;

- высвобождение работающего персонала;

- повышение качества выпускаемой продукции.

Поэтому эффективность предприятий можно повысить:

- совершенствованием структуры технологических процессов с целью повысить их управляемость и качество;

- уменьшением влияния вероятностных изменений промежуточных технологических процессов на изменение выходных параметров [4];

- увеличением объемов обрабатываемой информации с целью уменьшить неупорядоченность технологических процессов.

Понятие технической эффективности базируется на классическом рассмотрении производственной системы как «черного ящика», действия которого направлены на 
оптимальное достижение своих целей. В качестве источников технической эффективности рассматривается весь комплекс внутренних факторов, причем особое внимание уделяется совершенству системы управления, а также распределению нагрузки на элементы автоматики.

Рассмотрим основные причины неэффективности деятельности предприятия [14]:

1. Неэффективность вследствие неоптимального масштаба развернутости производства с избытком резервных элементов или их неупорядочностью (неэффективность по масштабу).

2. Неэффективность внутренних технологических процессов и объектов предприятия.

3. Неэффективность вследствие нерационального распределения потребляемых ресурсов и несоответствия качества (количества) продукции и спросу на нее.

4. Неэффективность вследствие недостаточно продуманного механизма управленческих решений, не полной реализации потенциала персонала и средств ACY.

Повышение эффективности работы предприятия осуществляется при помощи методов анализа структур предприятий, которые позволяют при прозрачности структуры и организации производства осуществлять точное и качественное слежение за состоянием объектов предприятия и управление ими. Рассмотрим некоторые из методов анализа структур предприятий в АСУП.

\section{4. Параметрические и непараметрические методы оценки сравнительной эффективности}

Параметрические и непараметрические методы были взяты нами из эконометрических подходов анализа эффективности организаций, но позволяют исследовать и производственные системы, так как рассматривают ресурсы и выпуск продукции. Полученные данные по показателям эффективности производств позволят принимать взвешенные решения и проводить планомерную политику повышения эффективности работы предприятий.

Проанализируем методы, отмеченные в [14-17]. Для начала рассмотрим непараметрические методы:

- метод DEA;

- метод свободного расположения оболочки (FDH);

- индексы производительности (РI).

\section{1. Метод DEA (Data Envelopment Analysis)}

Метод DEA основан на построении границы эффективности, которая является аналогом производственной функции для случая, когда выпуск не скалярный, а векторный. 
Граница эффективности имеет форму выпуклой оболочки или выпуклого конуса в пространстве входных и выходных переменных. Граница используется в качестве эталона для получения численного значения эффективности каждого из оцениваемых предприятий. Однако метод DEA имеет следующую особенность: он позволяет оценивать только относительную эффективность предприятий, то есть эффективность по сравнению друг с другом. Степень эффективности предприятий определяется их близостью к границе эффективности в многомерном пространстве входов и выходов. Способом построения границы эффективности является многократное решение задачи линейного программирования. Граница формируется как кусочно-линейная кривая, которая соединяет наиболее эффективные точки, тем самым, формируя выпуклую кривую производственных возможностей $[16,18,19]$.

\section{2. Метод свободного расположения оболочки}

Метод свободного расположения оболочки также называют «анализом оболочки данных со свободным размещением» (Free Disposable Hull Analysis). Данный подход, предложенный Депринсом, Симаром и Тулкенсом (Deprins, Simar, Tulkens [20, 21]) в 1984 году, представляет собой частный случай метода DEA, когда точки на линиях, соединяющих наиболее эффективные значения показателей, не включаются в границу эффективности. Таким образом, ни одно из предприятий не может достигнуть состояния максимальной эффективности. Основным отличием метода FDH является специфика построения границы эффективности. В основе метода DEA лежит возможность линейных комбинаций наборов входных и выходных переменных, что предполагает взаимозаменяемость ресурсов. В методе $\mathrm{FDH}$, наоборот, взаимозаменяемость отсутствует, применяется производственная функция леонтьевского типа. В результате чего граница эффективности имеет ступенчатый вид. Она образуется путем пересечения вертикальных и горизонтальных линий для каждой из рассматриваемых комбинаций входов и выходов $[16,20]$.

\section{3. Метод индексов производительности (Productivity indexes)}

Индекс - это относительная величина, показывающая, во сколько раз численное выражение уровня изучаемого явления в данных условиях отличается от уровня того же явления в других условиях. Различие условий может проявляться во времени (тогда говорят об индексах динамики), в пространстве (территориальные индексы), выборе в качестве базы сравнения какого-либо условного уровня, например планового показателя, уровня договорных обязательств и тому подобное. Они представляют собой простейшую оценку сравнительной производительности между двумя объектами. Идея индексов производительности (PI) основана на общей теории индексов. Индексы производительности представляют собой 
отношение суммы взвешенных выходных переменных к сумме взвешенных входных переменных. Примерами индексов производительности в анализе эффективности производственных объектов выступают индекс Малмквиста (Malmquist Productivity Index), используемый в паре с методом DEA; индекс Торнквиста (Tornqvist Productivity Index) и индекс Фишера (Fisher Productivity Index).

Индексы, которые рассчитываются по типу индексов физического объема, применимы при изучении наборов, состоящих как из разных объектов, так и из объектов одного и того же типа. Если совокупность неоднородна (например, совокупность производимых продуктов различного вида), то индекс физического объема - единственный способ показать динамику такой массы различных предметов, выражая еe через взвешивающий множитель (себестоимость, трудоемкость). Если совокупность состоит из объектов одного типа, то динамику этой массы можно показать непосредственно, сравнивая общее количество таких предметов в отчетном периоде с аналогичной величиной в базисном [17].

\section{4. Параметрические методы оценки эффективности}

Параметрические методы оценки эффективности опираются на известную спецификацию производственной функции, включают в себя метод стохастических границ, метод без спецификации распределения и метод густой границы. При использовании этих методов нельзя обойтись без вычисления основных параметров распределения (средних величин, дисперсий), поэтому они получили название параметрических методов.

Рассмотрим параметрические методы:

- метод стохастической границы;

- метод без спецификации распределения;

- метод густой границы.

\section{5. Стохастическая фронтирная модель (Stochastic Frontier Analysis, SFA)}

SFA-модель впервые была представлена Aigner, Lovell, Schmidt [22,23] и Meeusen, van den Broeck [22] в 1977 году. В основе метода стохастической границы лежит предположение о том, что эффективность распределена несимметрично, тогда как случайная ошибка подчиняется симметричному распределению. Как правило, в качестве закона распределения принимается стандартное нормальное распределение. Особенностями SFA-модели являются построение границы эффективности на основе заданной спецификации ее функциональной формы и непосредственное включение в модель случайного компонента. 


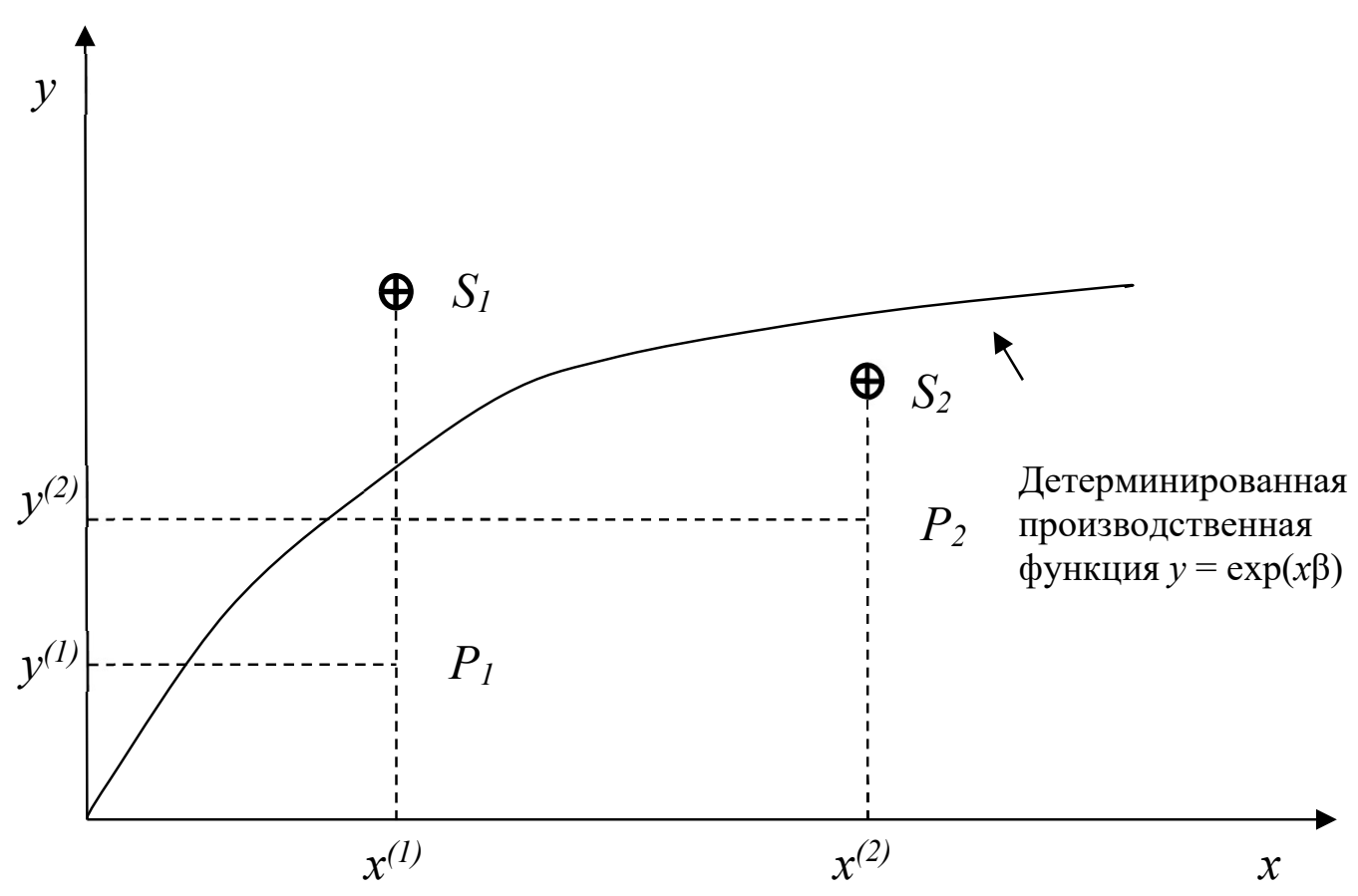

Рисунок 2. Стохастическая производственная функция.

SFA-модель была параметризована [24] в форме производственной функции КоббаДугласа:

$$
\ln \left(y^{(i)}\right)=x^{(i)} \beta+v^{(i)}-u^{(i)}, \quad i=1,2, \ldots, N
$$

где $N$ - количество рассматриваемых объектов; $\ln \left(y^{(i)}\right)$ - натуральный логарифм числового значения выпуска $i$ - го объекта; $x^{(i)}$ - вектор размерностью $(K+1)$, чей первый элемент равняется 1 , а все последующие элементы являются натуральными логарифмами значений количества используемых $i-$ м объектом $K$ ресурсов; $\beta=\left(\beta_{0}, \beta_{1}, \ldots, \beta_{K}\right)$ - вектор неизвестных параметров, подлежащих оценке; $v^{(i)}$ - случайная ошибка, предназначенная для измерения значения воздействия на значение переменной выпуска таких случайных факторов, как погода, забастовки, удача и тому подобное, а также общего воздействия неучтенных ресурсов переменных производственной функции; $u^{(i)}$ - имеющая положительное значение случайная переменная, связанная с технической неэффективностью производств в конкретной отрасли.

Эйгнер, Ловелл и Шмидт [22] предположили, что $v^{(i)}$ являются независимыми случайными переменными с одинаковым нормальным распределением с нулевым средним значением и постоянной вариацией. Они независимы от $u^{(i)}$, которые предполагались как независимые случайные переменные с одинаковым экспоненциальным или усеченным нормальным распределением. В такой модели значения выпуска ограничены стохастической переменной $\exp \left(x^{(\mathrm{i})} \beta+v^{(i)}\right)$. При этом случайная ошибка $v^{(i)}$ может принимать как положительные, так и отрицательные значения. Таким образом, граница производственных 


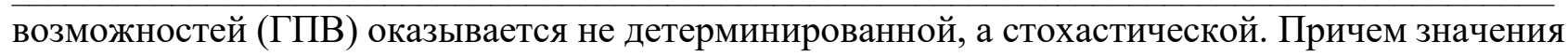
выпусков продукции варьируются вокруг детерминированной части рассматриваемой модели: $\exp \left(x^{(i)} \beta\right)$.

Основные особенности модели оценки стохастических ГПВ могут быть проиллюстрированы на двухмерном графике рисунок 2. Детерминированная составляющая модели изображена, исходя из предположения об уменьшающейся отдаче от масштаба. На графике представлены используемые ресурсы и выпуск для двух организаций, имеющих одинаковые технологии производства [25]. Организация 1 использует $x^{(i)}$ ресурсов для выпуска $y^{(1)}$ продукции. Указанное значение отмечено точкой $P_{l}$. Значение выпуска, соответствующее стохастической границе, отмечено точкой $S_{l}$. Оно находится выше производственной функции, так как случайная ошибка $v^{(1)}$ имеет положительное значение. Для фирмы 2 такая точка находится ниже производственной функции по причине отрицательного значения случайной ошибки $v^{(2)}$. Очевидно, что значения выпуска, лежащие на стохастической ГПВ, не наблюдаемы, так как не наблюдаемы соответствующие им случайные ошибки, $v^{(1)}$ и $v^{(2)}$. Тем не менее, детерминированная составляющая рассматриваемой модели лежит именно между этими значениями выпуска. Наблюдаемое значение выпуска фирмы может быть и выше детерминированной составляющей, если случайная ошибка (v) превышает показатель неэффективности для этой фирмы (u) [22]. Показателем неэффективности принимается условная средняя или мода распределения.

Метод SFA сначала разрабатывался в конце 1970-х гг. как абстрактная эконометрическая модель, носящая универсальный характер [15]. Стохастическая фронтирная модель включает в себя два компонента случайной переменной — симметричный, отражающий влияние случайных факторов, и строго неположительный, отражающий эффективность работы производства. Закон распределения случайного компонента эффективности выбирается исследователем и задается при спецификации модели. Одним из базовых предположений стохастической фронтирной модели является наличие общей производственной границы для всех объектов выборки. В случае нарушения данного предположения форма и положение границы (а как следствие и значения эффективностей работы всех объектов выборки) могут быть определены неправильно. Поэтому для корректной спецификации модели необходим предварительный анализ выборки на наличие выбросов $[14,26]$.

\section{6. Метод без спецификации распределения (distribution free approach, DFA)}

DFA-метод предполагает наличие некоторой средней эффективности для каждого предприятия за определенный период времени. Данный метод был предложен Бергером в 
1993 году [27]. В целом, метод DFA повторяет метод SFA, однако его особенность заключается в том, что оценка эффективности не может быть отделена от случайной ошибки, отделение эффективности от случайной компоненты производится иным способом. Предполагается, что неэффективность постоянна во времени, а средняя величина случайной ошибки за период стремится к нулю. Данная статистическая модель также основана на вероятностном подходе к определению границы и требует спецификации функционального вида границы эффективности. В отличие от стохастического фронтирного анализа, данный подход не требует строгих предположений о законе распределения эффективностей. Таким образом, данная модель строится на основе панельных данных и является модификацией модели со случайными эффектами [14].

\section{7. Метод густой границы}

Еще одним параметрическим методом, редко используемым в анализе производств, является метод густой (плотной, широкой) границы (Thick Frontier Approach, TFA), разработанный А. Бергером и Д. Хэмфри в 1991 году [27]. Метод ТFА основан на выделении из всей выборки двух квартилей - с наиболее высокими и наиболее низкими показателями эффективности. В рамках метода определяется функциональная форма зависимости издержек и прибыли от входных параметров. Предполагается, что отклонения в прогнозных значениях между наивысшим и самым низким квартилями представляет собой показатель эффективности. Метод TFA не предназначен для оценки эффективности отдельного предприятия, а нацелен на анализ общего уровня эффективности в выборке. Стохастическая модель на основе широкой границы использует только предположение о функциональной форме границы, не накладывая дополнительных ограничений на распределения эффективностей и случайной компоненты.

\section{5. Сравнительный анализ и обоснование выбора метода}

Для обоснования подходящего метода модельно-алгоритмической основы АСУП для оценки эффективности предприятий, сравним особенности рассмотренных методов. В таблице 1 приведем сравнительную характеристику по выделенным критериям параметрических методов.

Таблица 1. Сравнительная характеристика параметрических методов.

\begin{tabular}{|c|c|c|c|}
\hline Методы & $\begin{array}{l}\text { Особенности } \\
\text { Случайная ошибка }\end{array}$ & $\begin{array}{l}\text { Определение границы } \\
\text { распределения } \\
\text { эффективности }\end{array}$ & Закон распределения \\
\hline
\end{tabular}




\begin{tabular}{|c|c|c|c|}
\hline SFA & $\begin{array}{l}\text { Случайная ошибка } \\
\text { подчиняется } \\
\text { симметричному } \\
\text { распределению }\end{array}$ & $\begin{array}{l}\text { Эффективность } \\
\text { распределена } \\
\text { несимметрично, закон } \\
\text { распределения } \\
\text { случайного компонента } \\
\text { эффективности } \\
\text { выбирается } \\
\text { исследователем и } \\
\text { задается при } \\
\text { спецификации модели }\end{array}$ & $\begin{array}{l}\text { Стандартное } \\
\text { нормальное } \\
\text { распределение }\end{array}$ \\
\hline DFA & $\begin{array}{l}\text { Средняя величина } \\
\text { случайной ошибки за } \\
\text { период стремится к нулю }\end{array}$ & $\begin{array}{l}\text { Оценка эффективности } \\
\text { не может быть отделена } \\
\text { от случайной ошибки, } \\
\text { отделение } \\
\text { неэффективности от } \\
\text { случайной компоненты } \\
\text { производится иным } \\
\text { способом }\end{array}$ & $\begin{array}{l}\text { Требует строгих } \\
\text { предположений о } \\
\text { законе распределения } \\
\text { эффективностей. } \\
\text { Строится на основе } \\
\text { панельных данных и } \\
\text { является } \\
\text { модификацией модели } \\
\text { со случайными } \\
\text { эффектами }\end{array}$ \\
\hline TFA & $\begin{array}{l}\text { Отклонения от } \\
\text { прогнозных значений } \\
\text { внутри наивысшего и } \\
\text { самого низкого } \\
\text { квартилей представляет } \\
\text { собой случайную } \\
\text { ошибку }\end{array}$ & $\begin{array}{l}\text { Отклонения в } \\
\text { прогнозных значениях } \\
\text { между наивысшим и } \\
\text { самым низким } \\
\text { квартилями представляет } \\
\text { собой показатель } \\
\text { эффективности }\end{array}$ & $\begin{array}{l}\text { Предположение о } \\
\text { функциональной } \\
\text { форме границы }\end{array}$ \\
\hline
\end{tabular}

Из описания параметрических методов и их сравнительной характеристики можно сделать выводы:

- Метод фронтирной границы требует предварительного выбора закона распределения случайной составляющей эффективности и соответственно не может быть определен наверняка, а только с определенной долей вероятности, поэтому выбросы в показателях не удается полностью исключить. Метод SFA хорошо проработан только для случаев с одним выпускаемым продуктом или одним потребляемым ресурсом.

- Метод без спецификации распределения требует базового предположения о постоянном значении эффективности во времени. На практике данное предположение нарушается даже при условии сохранения организационных принципов работы предприятия из-за влияния научно-технического прогресса, изменений законодательной базы и экономических циклов. А также обязательное требование к наличию панельных данных (для симметричности влияния 
случайного компонента необходима достаточно длинная панель за длительный промежуток времени) [14].

- Метод густой границы не может предоставить индивидуальные значения эффективности для каждой организации, только возможность расчета средней эффективности по выборке.

Преимуществами параметрических (SFA, DFA, TFA) методов являются:

- В методах учтена возможность случайных ошибок, например, из-за ошибок построения границы или неверной отчетности. Неправильное измерение эффективности работы одного предприятия не влечет за собой смещение оценок остальных [15].

- Учитывают такую характеристику как стохастичность, все они дают оценку эффективности, а не ее строгое вычисление.

- Не требуется применение дополнительных методик (например, bootstrap [28]) для тестирования гипотез о значимости полученных оценок и влиянии различных факторов.

- Уменьшение влияния отдельных наблюдений (выбросов).

Преимущества непараметрических (DEA, FDH, PI) методов перед параметрическими:

- Отсутствие изначальных предположений о форме границы эффективности, граница представляется в виде произвольной ломаной.

- Отсутствие предположений о распределении эффективности работы организаций. Исключение составляет обязательность существования предприятий со стопроцентной эффективностью, которые и определяют вершины ломаной.

- Предоставляют точное вычисление показателя эффективности для каждого объекта.

- Возможность вычисления показателей объектов для большого количества входов и выходов (DEA).

Приведем в таблице 2 сравнительные характеристики непараметрических методов.

Таблица 2. Сравнительная характеристика непараметрических методов.

\begin{tabular}{llll}
\hline Методы & $\begin{array}{l}\text { Особенности } \\
\text { Построение } \\
\text { производственной } \\
\text { границы }\end{array}$ & $\begin{array}{l}\text { Взаимозаменяемость } \\
\text { входов и выходов }\end{array}$ & $\begin{array}{l}\text { Вид производственной } \\
\text { границы }\end{array}$ \\
\hline DEA & $\begin{array}{l}\text { Строится по матрице } \\
\text { входов и выходов }\end{array}$ & $\begin{array}{l}\text { Предполагает } \\
\text { перестановку входов и } \\
\text { выходов }\end{array}$ & $\begin{array}{l}\text { Граница формируется } \\
\text { как кусочно-линейная } \\
\text { кривая, которая }\end{array}$
\end{tabular}




\begin{tabular}{llll}
\hline & & & $\begin{array}{l}\text { соединяет наиболее } \\
\text { эффективные точки }\end{array}$ \\
FDH & Граница эффективности & Взаимозаменяемость & $\begin{array}{l}\text { Граница формируется } \\
\text { входов и выходов }\end{array}$ \\
& в виде ступенчатой \\
& Линейная граница, & отсутствует & ломанной \\
PI & взаимозаменяемость & Показывает отношения \\
& данным строится по & возможна между двумя & двух объектов в разные \\
& временных периодов & объектами & временные \\
\hline
\end{tabular}

Как следствие, непараметрические методы основываются на следующих предположениях [14]:

- отсутствие случайных факторов, которые могут влиять на эффективность работы предприятия;

- отсутствие ошибок при построении границы;

- совершенство отчетности, определяемой техническими средствами.

Из приведенных описаний и сравнительных характеристик, можно сделать следующие выводы:

- Метод оболочки данных (DEA) предоставляет измерение показателя эффективности при векторном входе и выходе, что позволяет подсчитывать показатели эффективности, учитывая множественные факторы и сравнивая большое количество объектов.

- Метод свободного расположения (FDH) оболочки не предполагает наличия предприятий с максимальной эффективностью и оценивает объекты, исходя из невозможности замены одних ресурсов другими, что суживает применение модели при оценке объектов в расширенной совокупности.

- Метод индексов производительности (PI) используется в основном для определения сравнительных показателей эффективности предприятий, рассматриваемых в определенный промежуток времени.

Необходимость применения непараметрических методов, обладающих свойствами анализа случайной ошибки и определения распределения эффективности по объектам выборки, отпадает. Так как мы работаем с измерительной информацией, на основе которой строятся показали входов и выходов предприятий, и которая определяется техническими средствами с достаточно высокой долей точности. Погрешности в точности получаемой информации ложатся на обслуживающий эти средства персонал.

Среди непараметрических методов выделим метод DEA и отметим необходимость применения его для модельно-алгоритмического обеспечения подсистем оперативного 
мониторинга в АСУП [29]. Значения показателя могут колебаться в интервале от 0 до 100\%, причем стопроцентную эффективность имеют предприятия, находящиеся на границе эффективности. К преимуществам данного подхода относятся объективность и воспроизводимость результатов. Построение границы эффективности и расчет индивидуальных значений эффективностей объектов производится с использованием четких математических моделей. Возможно включение в модель одновременно любого количества факторов, влияющих на эффективность, и определение значимости того или иного фактора. В результате имеется возможность сравнения предприятий на основе рассчитанных значений эффективностей [14, 28, 29].

\section{6. Заключение}

В работе приводится понятие автоматизированной системы управления предприятием, a также структура, некоторые особенности моделирования и понятие эффективности предприятия, как одной из целей его моделирования. В результате выделены основные задачи и функции системы, заключающиеся в обеспечении эффективного функционирования производства и поддержании работоспособности составляющих его модулей и объектов.

Существующие методы анализа структур и функционирования предприятий обладают рядом свойств, позволяющих повысить эффективность функционирования предприятий за счет анализа и синтеза их структуры. Известная методология «IDEF0», как наиболее приближенная к оценке предприятий, позволяет анализировать как структуру, так и способы взаимодействия объектов, входящих в производственную систему. Отдельно стоит выделить методы, основанные на технологиях «BMS» и «CASE», которые сочетают управление с производственными и организационными процессами, учитывая документооборот в управлении предприятием. Построение унифицированных структурных моделей технологических операций по «Методу анализа процесса» позволяет повысить управляемость отдельных объектов за счет прозрачности и унификации производства.

Аналитический обзор внешних и внутренних факторов по методу «SWOT-анализа» и обзор эталонных предприятий по методу «Бенчмаркинга» строят более полную картину работы производства. В логической основе методов «Тагутти» и «Функциональностоимостного анализа» лежит оптимизация производства и процессов, протекающих в нем, с целью повышения качества выпускаемой продукции и отладки связей между объектами управления в предприятии. Дополняют данные методы «Value stream mapping» и «Диаграммы Ишикавы», позволяющие оценивать структуру, находить причины снижения эффективности производств, и оптимизировать процесс создания промышленных продуктов по времени или обеспечить более оперативный отклик производства на изменяющиеся внешние условия. 
Моделирование функциональных связей и структур предприятий совместно с их системами управления позволяет построить подсистему анализа эффективности технологических процессов и производств в АСУ предприятием для повышения прозрачности работы и управляемости производства. Это приводит к повышению оперативности работы АСУП. Анализ сравнительной эффективности является достаточно важной составляющей процессов функционирования производства, более того, он должен осуществляться оперативно. Это позволит отлаживать работу предприятия буквально на первоначальных этапах снижения эффективности его функционирования. Поэтому важной задачей является поиск математической основы анализа эффективности предприятий.

В рамках данного обзора проанализированы параметрические и непараметрические методы оценки эффективности производственных объектов. Основными отличиями параметрических методов является эконометрическая оценка параметрических функций при исследовании взаимодействия затрат и ресурсов. Существенное внимание уделено непараметрическому методу DEA, который в силу своих характеристик, обладает рядом преимуществ и особенностей. Позволяет получать комплексный показатель эффективности для каждого предприятия и предполагает выдачу рекомендаций по повышению их эффективности, а также может учитывать множественные входные и выходные переменные. Эти особенности метода DEA позволяют успешно применить его при определении показателя сравнительной эффективности предприятий.

Проведенные исследования наметили необходимость решения следующих научнопрактических задач:

- дальнейшее развитие метода DEA и разработка его модификаций с целью учета дополнительных факторов при осуществлении оценки сравнительной эффективности работы предприятий различного типа;

- на основе рассмотренных методов оценки функционирования предприятий, реализовать способ анализа структуры предприятий;

- разработать методическое и алгоритмическое обеспечение оперативных подсистем мониторинга сравнительной эффективности технологических процессов и производств.

\section{Список литературы}

[1] Юдин, Д.Б. Математические методы оптимизации устройств и алгоритмов АСУ / Д.Б. Юдин, А.П. Горяшко, А.С. Немировский, под ред. Ю.В. Асафьева, В.А. Шабалина. - М.: Радио и связь, 1982. - 288 с.

[2] Гаспарский, В. Праксеологический анализ проектно-конструкторских разработок: Пер. 
польск / В. Гаспарский, под ред. А.И. Половникова. - М.: Мир, 1978. - 97 с.

[3] Новожилов, А.А. Модифицированный метод оценки сравнительной эффективности предприятий в подсистеме мониторинга АСУП: диссертация ... кандидата технических наук: 05.13.06 / А.А. Новожилов; [Место защиты: Сиб. аэрокосм. акад. им. акад. М.Ф. Решетнева]. - Красноярск, 2011.- 161 с., ил. РГБ ОД, 61 12-5/821.

[4] Вальков, В.М. Автоматизированные системы управления технологическими процессами. - 3-е изд., перераб. и доп. / В.М. Вальков, В.Е. Вершин. - Л.: Политехника, 1991. - 269 с.

[5] Гусаров, В.М. Статистика: Учеб. пособие для вузов / В.М. Гусаров. - М.: ЮНИТИДАНА, 2003. - 463 с.

[6] Зенюткин, Н. О способах формирования информационных структур для моделирования объектов, сред и процессов / Н. Зенюткин, Д. Ковалев, Е. Туев, Е. Туева // Современные инновации, системы и технологии - Modern Innovations, Systems and Technologies. - 2021. - № 1(1). - C. 10-22. https://doi.org/10.47813/2782-2818-2021-1-1$10-22$.

[7] Данильченко, И.А. Проектирование АСУП на основе типовых решений / И.А. Данильченко, А.С. Армягов, В.А. Егорова. - М.: Статистика, 1977. - 213 с.

[8] Охтелев, М.Ю. Интеллектуальные технологии мониторинга и управления структурной динамикой сложных технических объектов / М.Ю. Охтелев, Б.В. Соколов, Р.М. Юсупов. - М.: Наука, 2006. - 410 с.

[9] Биличенко В.В. Многоступенчатость организационно-технического развития производственных систем на автомобильном транспорте / В.В. Биличенко, С.О. Романюк. - М.: Машиностроение и транспорт, 2009. - №1. - 1-5 с.

[10] Р 50.1.028-2001 Информационные технологии поддержки жизненного цикла продукции. Методология функционального моделирования. - М.: Изд-во стандартов, 2001.

[11] Альпидовская, М.Л. и др. Макроэкономика: учебник / М.Л. Альпидовская и др. - Ростовна-Дону: Феникс, 2017. - 412 с.

[12] Реброва, Т.И. Мероприятия по эффективности производства предприятий легкой промышленности / Т.И. Реброва. Компьютерные технологии при моделировании, в управлении и экономике: Сборник научных трудов. - Харьков: ХАИ, 2004. - 400$405 \mathrm{c}$.

[13] Федоров, Ю.Н. Справочник инженера по АСУТП: Проектирование и разработка: учеб.прак.пос / Ю.Н. Федоров. - М.: Инфра - инженерия, 2008. - 928 с.

[14] Павлюк, Д.В. Статистическое исследование эффективности деятельности банков в Российской Федерации: дис. ... канд. эк. наук / Д.В. Павлюк. - М. 2005. - 59-64 с. 
[15] Моисеев, С.Р. Аналитический отчет. Эффективность российских банков. / С.Р. Моисеев. М: Изд-во МФПА, 2007. - 20 с.

[16] Новожилов, А.А. Анализ эффективности организационно-технологических комплексов предприятий / И.В. Ковалев, А.А. Новожилов, Т.А. Рукавицына // Системы управления и информационные технологии. - 2010. - 4(42). - С. 36-39.

[17] Иванов, Ю.Н. Экономическая статистика: учебник / под ред. Ю.Н. Иванова. - 5-е изд., перераб. и доп. - Москва: ИНФРА-М, 2019. - 584 с.

[18] Cooper, W.W. Handbook on Data Envelopment Analysis / W.W. Cooper, L.M. Seiford, J. Zhu (Eds.). - Boston: Kluwer Academic Publishers, 2004. - 608 p.

[19] Cooper, W. Data Envelopment Analysis. A Comprehensive Text with Models, Applications, References and DEA-Solver Software Second Edition / W. Cooper, Lawrence M. Seiford, K. Tone. - Springer Science+Business Media, LLC, 2007.

[20] Afonso, A., Public Sector Efficiency: An International Comparison / L. Schuknecht, V. Tanzi // European Central Bank Working Paper Series. - 2003. - No 242. - P. 18-23.

[21] Simar, L. Stochastic FDH/DEA estimators for frontier analysis / L. Simar, V. Zelenyuk // Springer Science+Business Media, 2010. - P.350-371.

[22] Aigner, D.J. Formulation and estimation of stochastic frontier production function models / D.J. Aigner, C.A.K. Lovell, P. Schmidt // Journal of Econometrics. - 1977. - 6(1). - P. 2137.

[23] Алескеров, Ф.Т. Анализ и оценка эффективности функционирования банков и банковских систем / Ф.Т. Алескеров, Ю.И. Мартынова, В.М. Солодков. - М.: Изд-во ГУ ВШЭ, 2009. - С.69-71.

[24] Гончаров, В. А. Методы оптимизации: учебное пособие для вузов / В. А. Гончаров. - Москва: Издательство Юрайт, 2020.

[25] Бертсекас, Д. Стохастическое оптимальное управление / Д. Бертсекас, С. Шрив. - М.: Наука, 1985. - 280 с.

[26] Coelli, T. An introduction to efficiency and productivity analysis / T. Coelli, D.S. Prasada Rao, G.E. Battese. - Boston: Kluwer Academic Publishers, 1998.

[27] Berger, A. The efficiency of bank branches / A. Berger, J. Leusner, J. Mingo // Journal of Monetary Economics. - 1997. - № 40. - P.141-162.

[28] Моргунов, Е.П. Многомерная классификация на основе аналитического метода оценки эффективности сложных систем: дисс...канд.техн.наук. - Красноярск, 2003.

[29] Туев, Е. В. Реализация мониторинга эффективности предприятий с помощью специальной подсистемы АСУП./ Е.В. Туев, М. Козлова, О. Ольшевская // Современные инновации, системы и технологии - Modern Innovations, Systems and 
Modern Innovations, Systems and Technologies, 2021, 1(3)

Technologies. - 2021. - 1(2). - 34-45. https://doi.org/10.47813/2782-2818-2021-1-2-34-45. 